\title{
Coupled fixed-point theorems for contraction mapping induced by cone ball-metric in partially ordered spaces
}

\author{
Wutiphol Sintunavarat ${ }^{1}$, Yeol Je $\mathrm{Cho}^{2^{*}}$ and Poom Kumam ${ }^{1 *}$
}

"Correspondence: yjcho@gnu.ac.kr; poom.kum@kmutt.ac.th 'Department of Mathematics, Faculty of Science, King Mongkut's University of Technology Thonburi (KMUTT), Bangkok, 10140, Thailand ${ }^{2}$ Department of Mathematics Education and the RINS, Gyeongsang National University, Chinju, 660-701, Korea

\begin{abstract}
Recently, Chen et al. (Appl. Math. Lett. 25:692-697, 2012) introduced the concept of the cone ball-metric and studied the common fixed-point theorems for the stronger Meir-Keeler cone-type function in cone ball-metric spaces. The purpose of this paper is to establish the coupled fixed-point theorems for nonlinear contraction mappings, which have a mixed monotone property by using the cone ball-metric. Also, we give some examples to validate our main results. At the end of this paper, we give an open problem for further investigation.
\end{abstract}

MSC: $47 \mathrm{H} 10 ; 54 \mathrm{H} 25$

Keywords: coupled fixed point; mixed monotone property; cone metric space; cone ball-metric space

\section{Introduction}

Fixed-point theory has been the most attractive topic to hundreds of researchers since 1922 with the celebrated Banach's contraction principle [11]. This principle provides a technique for solving a variety of applied problems in various branches of mathematics. Moreover, it provides the applications in many fields such as chemistry, biology, statistics, economics, computer science, and engineering. The Banach's contraction principle has been extended and improved by many mathematicians (see [7, 9, 13, 15, 24, 31-34] and others).

In 2004, the Banach's contraction principle was extended to metric spaces endowed with a partial ordering by Ran and Reurings [26]. Afterward, many generalizations and applications of the work of Ran and Reurings exist in the literature (see in [6, 17, 25]). For example, Nieto and Rodríguez-López [25] extended results of Ran and Reurings for nondecreasing mappings and studied a unique solution for a first-order ordinary differential equation with periodic boundary conditions.

In 2006, Bhaskar and Lakshmikantham [12] first introduced the concept of the mixed monotone property. Furthermore, they proved some coupled fixed-point theorems for mapping that satisfy the mixed monotone property and give some applications in the existence and uniqueness of a solution for a periodic boundary value problem

$$
u^{\prime}(t)=f(t, u(t)), \quad t \in[0, T], \quad u(0)=u(T)
$$

(0) 2012 Sintunavarat et al: licensee Springer. This is an Open Access article distributed under the terms of the Creative Commons Attribution License (http://creativecommons.org/licenses/by/2.0), which permits unrestricted use, distribution, and reproduction in any medium, provided the original work is properly cited. 
where the function $f$ satisfies certain conditions. Afterward, several authors studied and extended coupled fixed-point theorems of Bhaskar and Lakshmikantham [12] to different generalized condition (see, e.g., $[4,5,8,22,23,28,30,35,36]$ ).

On the other hand, the concept of cone metric spaces is a generalization of metric spaces, where each pair of points is assigned to a member of a real Banach space with a cone. This cone naturally induces a partial order in the Banach spaces. The concept of the cone metric space was reintroduced in the work of Huang and Zhang [18] where they also established the Banach's contraction mapping principle in this space. Afterward, several authors have studied fixed point and coupled fixed-point problems in cone metric spaces. Some of these works are noted in [1-3, 10, 20, 21, 29, 38]. Recently, Chen et al. [14] introduced the concept of cone ball-metric spaces and proved some fixed-point theorems in these spaces for mappings satisfying a contraction involving a stronger Meir-Keeler cone-type function.

Motivated by the interesting concept of cone ball-metric spaces of Chen et al. [14], in this paper, we establish some coupled fixed-point theorems for a contraction mapping induced by the cone ball-metric in partially ordered spaces and also study the condition claim of the uniqueness of a coupled fixed point. An open problem is also given at the end for further investigation.

\section{Preliminaries}

In this section, we shall recall some definitions and mathematical preliminaries.

Definition 2.1 Recall that a binary relation $\preceq$ on a nonempty set $X$ is said to be an order relation (and $X$ equipped with $\preceq$ is called a partially ordered set) if it satisfies the following three properties:

(i) reflexivity: $x \preceq x$ for all $x \in X$,

(ii) antisymmetry: $x \preceq y$ and $y \preceq x$ imply $x=y$,

(iii) transitivity: $x \preceq y$ and $y \preceq z$ imply $x \preceq z$.

Throughout this paper $(X, \preceq)$ denotes a partially ordered set. By $x \succeq y$ holds, we mean that $y \preceq x$ holds and by $x \prec y$ holds we mean that $x \preceq y$ holds, but $x \neq y$. If $(X, \preceq)$ is a partially ordered set and $f: X \rightarrow X$ is such that, for all $x, y \in X, x \preceq y$ implies $f(x) \preceq f(y)$, then a mapping $f$ is said to be nondecreasing. Similarly, a nonincreasing mapping is also defined.

Definition 2.2 ([12]) Let $(X, \preceq)$ be a partial ordered set and $F: X \times X \rightarrow X$ be a mapping. The mapping $F$ is said to has the mixed monotone property if $F$ is monotone nondecreasing in its first argument and is monotone nonincreasing in its second argument, that is, for any $x, y \in X$,

$$
x_{1}, x_{2} \in X, x_{1} \preceq x_{2} \quad \Longrightarrow \quad F\left(x_{1}, y\right) \preceq F\left(x_{2}, y\right)
$$

and

$$
y_{1}, y_{2} \in X, y_{1} \preceq y_{2} \quad \Longrightarrow \quad F\left(x, y_{1}\right) \succeq F\left(x, y_{2}\right) .
$$

Definition 2.3 ([12]) Let $X$ be a nonempty set. An element $(x, y) \in X \times X$ is called a coupled fixed point of the mapping $F: X \times X \rightarrow X$ if $x=F(x, y)$ and $y=F(y, x)$. 
Next, we give some notations and lemmas of cone metric spaces which are reintroduced by Huang and Zhang [18].

Let $E$ be a real Banach space and $0_{E}$ denote the zero element in $E$. A cone $P$ is a subset of $E$ such that

$\left(C_{1}\right) P$ is nonempty closed and $P \neq\left\{0_{E}\right\}$;

$\left(C_{2}\right)$ if $a, b$ are nonnegative real numbers and $x, y \in P$, then $a x+b y \in P$;

$\left(C_{3}\right) P \cap(-P)=\left\{0_{E}\right\}$.

For any cone $P \subset E$, the partial ordering $\leq_{E}$ with respect to $P$ defined by $x \leq_{E} y$ if and only if $y-x \in P$. We write $x<_{E} y$ to indicate that $x \leq_{E} y$, but $x \neq y$, while $x \ll y$ stands for $y-x \in \operatorname{int}(P)$, where int $(P)$ denotes the interior of $P$.

A cone $P$ is said to be normal if there is a number $K>0$ such that, for all $x, y \in E$,

$$
0_{E} \leq_{E} x \leq_{E} y \quad \Longrightarrow \quad\|x\| \leq K\|y\|
$$

The least positive number satisfying above is called the normal constant of $P$.

The cone $P$ is said to be regular if every increasing sequence which is bounded from above is convergent, that is, if $\left\{x_{n}\right\}$ is a sequence in $E$ such that

$$
x_{1} \leq_{E} x_{2} \leq_{E} \cdots \leq_{E} y
$$

for some $y \in E$, then there is $x \in E$ such that $\lim _{n \rightarrow \infty}\left\|x_{n}-x\right\|=0$. Equivalently, the cone $P$ is said to be regular if every decreasing sequence which is bounded from below is convergent. It is well known that a regular cone is a normal cone (see also [27]).

Remark 2.4 ([18]) (1) If $E$ be a real Banach space with a cone $P$ in $E$ and $a \leq_{E} k a$, where $a \in E$ and $0<k<1$, then $a=0_{E}$.

(2) If $c \in \operatorname{int}(P), 0_{E} \leq_{E} a_{n}$ and $a_{n} \rightarrow 0_{E}$, then there exists a positive integer $N$ such that $a_{n} \ll c$ for all $n \geq N$.

Lemma 2.5 ([21]) If E be a real Banach space with a cone P in E, then we have the following:

(1) If $0_{E} \leq_{E} x \leq_{E} y$ and $k$ is a nonnegative real number, then $0_{E} \leq_{E} k x \leq_{E} k y$.

(2) If $0_{E} \leq_{E} x_{n} \leq_{E} y_{n}$ for all $n \in \mathbb{N}$ and $x_{n} \rightarrow x, y_{n} \rightarrow y$ as $n \rightarrow \infty$, then $0_{E} \leq_{E} x \leq_{E} y$.

Lemma 2.6 ([19]) If E be a real Banach space with a cone P in E, then we have the following: for all $a, b, c \in E$,

(1) If $a \leq_{E} b$ and $b \ll c$, then $a \ll c$.

(2) If $a \ll b$ and $b \ll c$, then $a \ll c$.

Using the notation of a cone, we have following definitions of cone metric space.

Definition 2.7 ([18]) Let $X$ be a nonempty set and $E$ be a real Banach space equipped with the partial ordering $\leq_{E}$ with respect to the cone $P \subset E$. Suppose that the mapping $d: X \times X \rightarrow E$ satisfies the following conditions:

$\left(C M_{1}\right) 0_{E}<_{E} d(x, y)$ for all $x, y \in X$ with $x \neq y$ and $d(x, y)=0_{E}$ if and only if $x=y$; 
$\left(C M_{2}\right) d(x, y)=d(y, x)$ for all $x, y \in X$;

$\left(C M_{3}\right) d(x, y) \leq_{E} d(x, z)+d(z, y)$ for all $x, y, z \in X$.

Then $d$ is called a cone metric on $X$ and $(X, d)$ is called a cone metric space.

For other basic properties on a cone metric space, the reader can refer to [18].

Next, we give the concept of a cone ball-metric space introduced by Chen et al. [14] and its properties.

In the following, we always suppose that $E$ is a real Banach space endowed with a cone $P$ with the apex at the origin $0_{E}, \operatorname{int}(P) \neq \emptyset$ and a linear ordering $\leq_{E}$ with respect to $P$.

Definition 2.8 ([14] $)$ Let $(X, d)$ be a cone metric space. A cone ball-metric with respect to the cone metric $d$ is a function $\mathcal{B}: X \times X \times X \rightarrow E$ defined by

$$
\mathcal{B}(x, y, z)=\inf \left\{2 \gamma:\{x, y, z\} \subset B_{\gamma}\right\}
$$

where

$$
B_{\gamma}(x):=\{y \in X: d(x, y)<\gamma\}
$$

for $x \in X$ is a ball in $X$ with the center $x$ and radius $\gamma \gg 0_{E}$. The ordered pair $(X, \mathcal{B})$ is called a cone ball-metric space.

Proposition $2.9([14])$ If $(X, \mathcal{B})$ is a cone ball-metric space, then the following statements hold:

$\left(\mathcal{B}_{1}\right) \mathcal{B}(x, y, z)=0_{E}$ if $x=y=z$;

$\left(\mathcal{B}_{2}\right) \mathcal{B}(x, x, y)>0_{E}$ for all $x, y \in X$ with $x \neq y$;

$\left(\mathcal{B}_{3}\right) \mathcal{B}(x, x, y) \leq \mathcal{B}(x, y, z)$ for all $x, y, z \in X$

$\left(\mathcal{B}_{4}\right) \mathcal{B}(x, y, z)=\mathcal{B}(x, z, y)=\mathcal{B}(y, z, x)=\cdots$ (symmetry in all three variables);

$\left(\mathcal{B}_{5}\right) \mathcal{B}(x, y, z) \leq \mathcal{B}(x, w, w)+\mathcal{B}(w, y, z)$ for all $x, y, z, w \in X$ (rectangle inequality);

(B) $\mathcal{B}(x, x, y)=\mathcal{B}(x, y, y)$ for all $x, y \in X$.

Definition $2.10([14])$ Let $(X, \mathcal{B})$ be a cone ball-metric space and $\left\{x_{n}\right\}$ be a sequence in $X$. We say that $\left\{x_{n}\right\}$ is called:

(1) A Cauchy sequence if, for any $\epsilon \in E$ with $0_{E} \ll \epsilon$, there exists a positive integer $N$ such that $\mathcal{B}\left(x_{n}, x_{m}, x_{l}\right) \ll \epsilon$ for all $n, m, l>N$.

(2) A convergent sequence if, for any $\epsilon \in E$ with $0_{E} \ll \epsilon$, there exists a positive integer $N$ such that, for all $n, m>N, \mathcal{B}\left(x, x_{n}, x_{m}\right) \ll \epsilon$ for some $x \in X$. Here, $x$ is called the limit of the sequence $\left\{x_{n}\right\}$ and is denoted by $\lim _{n \rightarrow \infty} x_{n}$ or $x_{n} \rightarrow x$.

Remark 2.11 We can prove easily that $\left\{x_{n}\right\}$ is a Cauchy sequence if and only if, for any $\epsilon \in E$ with $0_{E} \ll \epsilon$, there exists a positive integer $N$ such that $\mathcal{B}\left(x_{n}, x_{m}, x_{m}\right) \ll \epsilon$ for all $n, m>N$.

Definition $2.12([14])$ A cone ball-metric space $(X, \mathcal{B})$ is said to be complete if every Cauchy sequence is convergent in $X$. 
Proposition $2.13([14])$ Let $(X, \mathcal{B})$ be a cone ball-metric space and $\left\{x_{n}\right\}$ be a sequence of points of $X$. Then the following are equivalent:

(1) $x_{n} \rightarrow x$ as $n \rightarrow \infty$.

(2) $\mathcal{B}\left(x_{n}, x_{n}, x\right) \rightarrow 0_{E}$ as $n \rightarrow \infty$.

(3) $\mathcal{B}\left(x_{n}, x, x\right) \rightarrow 0_{E}$ as $n \rightarrow \infty$.

(4) $\mathcal{B}\left(x_{n}, x_{m}, x\right) \rightarrow 0_{E}$ as $n, m \rightarrow \infty$.

Proposition 2.14 ([14]) Let $(X, \mathcal{B})$ be a cone ball-metric space, $\left\{x_{n}\right\}$ be a sequence of points of $X$ and $x, y \in X$. If $x_{n} \rightarrow x$ and $x_{n} \rightarrow y$ as $n \rightarrow \infty$, then $x=y$.

Proposition $2.15([14])$ Let $(X, \mathcal{B})$ be a cone ball-metric space and $\left\{x_{n}\right\},\left\{y_{m}\right\}$ and $\left\{z_{l}\right\}$ be tree sequences in $X$. If $x_{n} \rightarrow x, y_{m} \rightarrow y$, and $z_{l} \rightarrow z$ as $n, m, l \rightarrow \infty$, then $\mathcal{B}\left(x_{n}, y_{m}, z_{l}\right) \rightarrow$ $\mathcal{B}(x, y, z)$ as $n, m, l \rightarrow \infty$.

Definition 2.16 Let $(X, \mathcal{B})$ be a cone ball-metric space. A mapping $F: X \times X \rightarrow X$ is said to be continuous if for any two convergent sequences $\left\{x_{n}\right\}$ and $\left\{y_{n}\right\}$ converging to $x$ and $y$ in $X$, respectively, then $\left\{F\left(x_{n}, y_{n}\right)\right\}$ is convergent to $F(x, y)$.

\section{Existence of coupled fixed point in cone ball-metric spaces}

Let $\Delta$ denote the class of all functions $\delta:\left(\operatorname{int}(P) \cup\left\{0_{E}\right\}\right) \times\left(\operatorname{int}(P) \cup\left\{0_{E}\right\}\right) \rightarrow[0,1)$ which satisfies the following condition.

For any sequences $\left\{a_{n}\right\}$ and $\left\{b_{n}\right\} \operatorname{in} \operatorname{int}(P) \cup\left\{0_{E}\right\}$,

$$
\delta\left(a_{n}, b_{n}\right) \rightarrow 1 \quad \Longrightarrow \quad a_{n}, b_{n} \rightarrow 0_{E}
$$

Let $E=\mathbb{R}$ be a usual norm space with a cone $P=\{z \in \mathbb{R} \mid z \geq 0\}$ and $X=\mathbb{R}$ be a cone metric space with a metric $d(x, y)=|x-y|$ for all $x, y \in X$. The following are examples of the functions in $\Delta$ under above setting:

(1) $\delta_{1}(a, b)=k$, where $k \in[0,1)$.

(2) $\delta_{2}(a, b)=\left\{\begin{array}{ll}\frac{\ln (1+k a+l b)}{k a+l b} ; & a>0 \text { or } b>0, \\ r \in[0,1) ; & a=b=0,\end{array} \quad\right.$ where $k, l \in[0,1)$.

(3) $\delta_{3}(a, b)= \begin{cases}\frac{\ln (1+\max \{a, b\})}{\max \{a, b\}} ; & a>0 \text { or } b>0, \\ r \in[0,1) ; & a=b=0 .\end{cases}$

Next, we prove our main theorems.

Theorem 3.1 Let $(X, \preceq)$ be a partially ordered set and $\mathcal{B}$ be a cone ball-metric induced by the cone metric $d$ on $X$ with a regular cone $P$ such that $(X, \mathcal{B})$ is a complete cone ball-metric space. Let $F: X \times X \rightarrow X$ be a continuous mapping having the mixed monotone property on $X$. Suppose that there exists $\delta \in \Delta$ such that

$$
\begin{gathered}
\mathcal{B}(F(x, y), F(u, v), F(w, z))+\mathcal{B}(F(y, x), F(v, u), F(z, w)) \\
\quad \leq_{E} \delta(\mathcal{B}(x, u, w), \mathcal{B}(y, v, z))(\mathcal{B}(x, u, w)+\mathcal{B}(y, v, z))
\end{gathered}
$$

for all $x, y, z, u, v, w \in X$ for which $x \succeq u \succeq w$ and $y \preceq v \preceq z$. If there exists $x_{0}, y_{0} \in X$ such that

$$
x_{0} \preceq F\left(x_{0}, y_{0}\right) \quad \text { and } \quad y_{0} \succeq F\left(y_{0}, x_{0}\right) \text {, }
$$


then $F$ has a coupled fixed point, that is, there exist $x, y \in X$ such that

$$
x=F(x, y) \text { and } y=F(y, x) .
$$

Proof We construct two sequences $\left\{x_{n}\right\},\left\{y_{n}\right\}$ in $X$ such that, for all $n \geq 0$,

$$
x_{n+1}=F\left(x_{n}, y_{n}\right) \quad \text { and } \quad y_{n+1}=F\left(y_{n}, x_{n}\right) .
$$

First, by induction, we show that, for all $n \geq 0$,

$$
x_{n} \preceq x_{n+1} \quad \text { and } \quad y_{n} \succeq y_{n+1} .
$$

From $x_{0} \preceq F\left(x_{0}, y_{0}\right)=x_{1}$ and $y_{0} \succeq F\left(y_{0}, x_{0}\right) \succeq y_{1}$, in case $n=0$, (3.3) holds. Assume that (3.3) holds for some $n \geq 0$. Then we get

$$
x_{n} \preceq x_{n+1} \quad \text { and } \quad y_{n} \succeq y_{n+1} \text {. }
$$

Since $F$ has the mixed monotone property, it follows from (3.4) and (2.1) that

$$
F\left(x_{n}, y\right) \preceq F\left(x_{n+1}, y\right) \quad \text { and } \quad F\left(y_{n+1}, x\right) \preceq F\left(y_{n}, x\right)
$$

for all $x, y \in X$. From (3.4) and (2.2), we have

$$
F\left(y, x_{n}\right) \succeq F\left(y, x_{n+1}\right) \quad \text { and } \quad F\left(x, y_{n+1}\right) \succeq F\left(x, y_{n}\right) \text {, }
$$

for all $x, y \in X$.

If we take $y=y_{n}$ and $x=x_{n}$ in (3.5), we get

$$
x_{n+1}=F\left(x_{n}, y_{n}\right) \preceq F\left(x_{n+1}, y_{n}\right) \quad \text { and } \quad F\left(y_{n+1}, x_{n}\right) \preceq F\left(y_{n}, x_{n}\right)=y_{n+1} \text {. }
$$

If we take $y=y_{n+1}$ and $x=x_{n+1}$ in (3.6), we get

$$
F\left(y_{n+1}, x_{n}\right) \succeq F\left(y_{n+1}, x_{n+1}\right)=y_{n+2} \quad \text { and } \quad x_{n+2}=F\left(x_{n+1}, y_{n+1}\right) \succeq F\left(x_{n+1}, y_{n}\right) \text {. }
$$

From (3.7) and (3.8), we also have

$$
x_{n+1} \preceq x_{n+2} \quad \text { and } \quad y_{n+1} \succeq y_{n+2} \text {. }
$$

Consequently, by induction, we have (3.3) holds for all $n \geq 0$. This implies

$$
x_{0} \preceq x_{1} \preceq x_{2} \preceq \cdots \preceq x_{n} \preceq x_{n+1} \preceq \cdots
$$

and

$$
y_{0} \succeq y_{1} \succeq y_{2} \succeq \cdots \succeq y_{n} \succeq y_{n+1} \succeq \cdots .
$$


If there exists an integer number $n^{\star} \geq 0$ such that

$$
\mathcal{B}\left(x_{n^{\star}+1}, x_{n^{\star}+1}, x_{n^{\star}}\right)+\mathcal{B}\left(y_{n^{\star}+1}, y_{n^{\star}+1}, y_{n^{\star}}\right)=0_{E},
$$

then we have

$$
\mathcal{B}\left(x_{n^{\star}+1}, x_{n^{\star}+1}, x_{n^{\star}}\right)=\mathcal{B}\left(y_{n^{\star}+1}, y_{n^{\star}+1}, y_{n^{\star}}\right)=0_{E},
$$

which implies $x_{n^{\star}}=x_{n^{\star}+1}$ and $y_{n^{\star}}=y_{n^{\star}+1}$. Therefore, $x_{n^{\star}}=F\left(x_{n^{\star}}, y_{n^{\star}}\right)$ and $y_{n^{\star}}=F\left(y_{n^{\star}}, x_{n^{\star}}\right)$ and so $\left(x_{n^{\star}}, y_{n^{\star}}\right)$ is a coupled fixed point of $F$.

Now, we assume that $\mathcal{B}\left(x_{n+1}, x_{n+1}, x_{n}\right)+\mathcal{B}\left(y_{n+1}, y_{n+1}, y_{n}\right)>_{E} 0_{E}$ for all $n \geq 0$. Since $x_{n-1} \preceq x_{n}$ and $y_{n-1} \succeq y_{n}$ for all $n \in \mathbb{N}$, by (3.1) and (3.2), we have

$$
\begin{aligned}
\mathcal{B} & \left(x_{n+1}, x_{n+1}, x_{n}\right)+\mathcal{B}\left(y_{n+1}, y_{n+1}, y_{n}\right) \\
& =\mathcal{B}\left(F\left(x_{n}, y_{n}\right), F\left(x_{n}, y_{n}\right), F\left(x_{n-1}, y_{n-1}\right)\right)+\mathcal{B}\left(F\left(y_{n}, x_{n}\right), F\left(y_{n}, x_{n}\right), F\left(y_{n-1}, x_{n-1}\right)\right) \\
& \leq_{E} \delta\left(\mathcal{B}\left(x_{n}, x_{n}, x_{n-1},\right), \mathcal{B}\left(y_{n}, y_{n}, y_{n-1}\right)\right)\left[\mathcal{B}\left(x_{n}, x_{n}, x_{n-1},\right)+\mathcal{B}\left(y_{n}, y_{n}, y_{n-1}\right)\right] \\
& <_{E} \mathcal{B}\left(x_{n}, x_{n}, x_{n-1},\right)+\mathcal{B}\left(y_{n}, y_{n}, y_{n-1}\right) .
\end{aligned}
$$

So, we have the sequence $\left\{\mathcal{B}_{n}\right\}$ defined by $\mathcal{B}_{n}:=\mathcal{B}\left(x_{n}, x_{n}, x_{n-1}\right)+\mathcal{B}\left(y_{n}, y_{n}, y_{n-1}\right)$ is a decreasing sequence. Since $P$ is regular, there exists $\mathcal{B} \geq_{E} 0_{E}$ such that $\mathcal{B}_{n} \rightarrow \mathcal{B}$ as $n \rightarrow \infty$.

Next, we prove that $\mathcal{B}=0_{E}$. Suppose that $\mathcal{B}>_{E} 0_{E}$. From (3.12), letting $n \rightarrow \infty$, we have

$$
\delta\left(\mathcal{B}\left(x_{n}, x_{n}, x_{n-1}\right), \mathcal{B}\left(y_{n}, y_{n}, y_{n-1}\right)\right) \rightarrow 1
$$

Since $\delta \in \Delta$, we have $\mathcal{B}\left(x_{n}, x_{n}, x_{n-1}\right) \rightarrow 0_{E}$ and $\mathcal{B}\left(y_{n}, y_{n}, y_{n-1}\right) \rightarrow 0_{E}$ and then

$$
\mathcal{B}\left(x_{n}, x_{n}, x_{n-1}\right)+\mathcal{B}\left(y_{n}, y_{n}, y_{n-1}\right) \rightarrow 0_{E},
$$

which contradictions with $\mathcal{B}>_{E} 0_{E}$. Consequently, we must get

$$
\mathcal{B}\left(x_{n}, x_{n}, x_{n-1}\right)+\mathcal{B}\left(y_{n}, y_{n}, y_{n-1}\right) \rightarrow 0_{E}
$$

By the property $\left(\mathcal{B}_{6}\right)$, we have

$$
\mathcal{B}_{n}^{\prime}:=\mathcal{B}\left(x_{n}, x_{n-1}, x_{n-1}\right)+\mathcal{B}\left(y_{n}, y_{n-1}, y_{n-1}\right) \rightarrow 0_{E} .
$$

Now, we show that $\left\{x_{n}\right\}$ and $\left\{y_{n}\right\}$ are Cauchy sequences in cone ball-metric space $(X, \mathcal{B})$. Suppose on the contrary that at least one of $\left\{x_{n}\right\}$ and $\left\{y_{n}\right\}$ are not a Cauchy sequence in $(X, \mathcal{B})$. Then there exists $\epsilon \in E$ such that $0_{E} \ll \epsilon$ and sequences of positive integers $\{n(k)\}$ and $\{m(k)\}$ such that for all positive integers $k$,

$$
n(k)>m(k) \geq k
$$

and

$$
\mathcal{B}\left(x_{n(k)}, x_{m(k)}, x_{m(k)}\right)+\mathcal{B}\left(y_{n(k)}, y_{m(k)}, y_{m(k)}\right)>_{E} \epsilon .
$$


Further, for the integer $m(k)$, we can choose $n(k)$ is the smallest integer for which (3.15) holds. Then we have

$$
\mathcal{B}\left(x_{n(k)-1}, x_{m(k)}, x_{m(k)}\right)+\mathcal{B}\left(y_{n(k)-1}, y_{m(k)}, y_{m(k)}\right) \leq_{E} \epsilon .
$$

Using (3.15) and (3.16) and the rectangle inequality, we have

$$
\begin{array}{rl}
\epsilon<_{E} & r_{k}:=\mathcal{B}\left(x_{n(k)}, x_{m(k)}, x_{m(k)}\right)+\mathcal{B}\left(y_{n(k)}, y_{m(k)}, y_{m(k)}\right) \\
\leq_{E} & \mathcal{B}\left(x_{n(k)}, x_{n(k)-1}, x_{n(k)-1}\right)+\mathcal{B}\left(x_{n(k)-1}, x_{m(k)}, x_{m(k)}\right) \\
& +\mathcal{B}\left(y_{n(k)}, y_{n(k)-1}, y_{n(k)-1}\right)+\mathcal{B}\left(y_{n(k)-1}, y_{m(k)}, y_{m(k)}\right) \\
\leq_{E} & \mathcal{B}\left(x_{n(k)}, x_{n(k)-1}, x_{n(k)-1}\right)+\mathcal{B}\left(y_{n(k)}, y_{n(k)-1}, y_{n(k)-1}\right)+\epsilon .
\end{array}
$$

Letting $k \rightarrow \infty$, we have

$$
r_{k}=\mathcal{B}\left(x_{n(k)}, x_{m(k)}, x_{m(k)}\right)+\mathcal{B}\left(y_{n(k)}, y_{m(k)}, y_{m(k)}\right) \rightarrow \epsilon
$$

By the rectangle inequality, we get

$$
\begin{array}{rl}
r_{k}= & \mathcal{B}\left(x_{n(k)}, x_{m(k)}, x_{m(k)}\right)+\mathcal{B}\left(y_{n(k)}, y_{m(k)}, y_{m(k)}\right) \\
\leq_{E} & \mathcal{B}\left(x_{n(k)}, x_{n(k)+1}, x_{n(k)+1}\right)+\mathcal{B}\left(x_{n(k)+1}, x_{m(k)+1}, x_{m(k)+1}\right)+\mathcal{B}\left(x_{m(k)+1}, x_{m(k)}, x_{m(k)}\right) \\
& +\mathcal{B}\left(y_{n(k)}, y_{n(k)+1}, y_{n(k)+1}\right)+\mathcal{B}\left(y_{n(k)+1}, y_{m(k)+1}, y_{m(k)+1}\right)+\mathcal{B}\left(y_{m(k)+1}, y_{m(k)}, y_{m(k)}\right) \\
= & {\left[\mathcal{B}\left(x_{n(k)+1}, x_{m(k)+1}, x_{m(k)+1}\right)+\mathcal{B}\left(y_{n(k)+1}, y_{m(k)+1}, y_{m(k)+1}\right)\right]} \\
& +\left[\mathcal{B}\left(x_{n(k)}, x_{n(k)+1}, x_{n(k)+1}\right)+\mathcal{B}\left(y_{n(k)}, y_{n(k)+1}, y_{n(k)+1}\right)\right] \\
& +\left[\mathcal{B}\left(x_{m(k)+1}, x_{m(k)}, x_{m(k)}\right)+\mathcal{B}\left(y_{m(k)+1}, y_{m(k)}, y_{m(k)}\right)\right] \\
= & {\left[\mathcal{B}\left(x_{n(k)+1}, x_{m(k)+1}, x_{m(k)+1}\right)+\mathcal{B}\left(y_{n(k)+1}, y_{m(k)+1}, y_{m(k)+1}\right)\right]+\mathcal{B}_{n(k)+1}+\mathcal{B}_{m(k)+1}^{\prime}} \\
= & {\left[\mathcal{B}\left(x_{m(k)+1}, x_{m(k)+1}, x_{n(k)+1}\right)+\mathcal{B}\left(y_{m(k)+1}, y_{m(k)+1}, y_{n(k)+1}\right)\right]+\mathcal{B}_{n(k)+1}+\mathcal{B}_{m(k)+1}^{\prime}} \\
= & {\left[\mathcal{B}\left(F\left(x_{m(k)}, y_{m(k)}\right), F\left(x_{m(k)}, y_{m(k)}\right), F\left(x_{n(k)}, y_{n(k)}\right)\right)\right.} \\
& \left.+\mathcal{B}\left(F\left(y_{m(k)}, x_{m(k)}\right), F\left(y_{m(k)}, x_{m(k)}\right), F\left(y_{n(k)}, x_{n(k)}\right)\right)\right]+\mathcal{B}_{n(k)+1}+\mathcal{B}_{m(k)+1}^{\prime} \\
\leq & \delta\left(\mathcal{B}\left(x_{m(k)}, x_{m(k)}, x_{n(k)}\right), \mathcal{B}\left(y_{m(k)}, y_{m(k)}, y_{n(k)}\right)\right) \\
& \times\left(\mathcal{B}\left(x_{m(k)}, x_{m(k)}, x_{n(k)}\right)+\mathcal{B}\left(y_{m(k)}, y_{m(k)}, y_{n(k)}\right)\right)+\mathcal{B}_{n(k)+1}+\mathcal{B}_{m(k)+1}^{\prime} \\
=\delta & \left.\mathcal{B}\left(x_{n(k)}, x_{m(k)}, x_{m(k)}\right), \mathcal{B}\left(y_{n(k)}, y_{m(k)}, y_{m(k)}\right)\right) r_{k}+\mathcal{B}_{n(k)+1}+\mathcal{B}_{m(k)+1}^{\prime} \\
<E & r_{k}+\mathcal{B}_{n(k)+1}+\mathcal{B}_{m(k)+1}^{\prime} \cdot \\
&
\end{array}
$$

Taking $k \rightarrow \infty$ and using (3.13), (3.14), and (3.17), we get

$$
\delta\left(\mathcal{B}\left(x_{n(k)}, x_{m(k)}, x_{m(k)}\right), \mathcal{B}\left(y_{n(k)}, y_{m(k)}, y_{m(k)}\right)\right) \rightarrow 1
$$

Since $\delta \in \Delta$, we have $\mathcal{B}\left(x_{n(k)}, x_{m(k)}, x_{m(k)}\right) \rightarrow 0_{E}$ and $\mathcal{B}\left(y_{n(k)}, y_{m(k)}, y_{m(k)}\right) \rightarrow 0_{E}$, that is,

$$
\mathcal{B}\left(x_{n(k)}, x_{m(k)}, x_{m(k)}\right)+\mathcal{B}\left(y_{n(k)}, y_{m(k)}, y_{m(k)}\right) \rightarrow 0_{E},
$$


which contradictions with (3.17). Therefore, $\left\{x_{n}\right\}$ and $\left\{y_{n}\right\}$ are Cauchy sequences in $(X, \mathcal{B})$. Since $X$ complete, we get $x_{n} \rightarrow x$ and $y_{n} \rightarrow y$ as $n \rightarrow \infty$ for some $x, y \in X$.

Finally, we prove that $(x, y)$ is a coupled fixed point of $F$. Since $F$ is a continuous, taking $n \rightarrow \infty$ in (3.2), we get

$$
x=\lim _{n \rightarrow \infty} x_{n+1}=\lim _{n \rightarrow \infty} F\left(x_{n}, y_{n}\right)=F\left(\lim _{n \rightarrow \infty} x_{n}, \lim _{n \rightarrow \infty} y_{n}\right)=F(x, y)
$$

and

$$
y=\lim _{n \rightarrow \infty} y_{n+1}=\lim _{n \rightarrow \infty} F\left(y_{n}, x_{n}\right)=F\left(\lim _{n \rightarrow \infty} y_{n}, \lim _{n \rightarrow \infty} x_{n}\right)=F(y, x) .
$$

Therefore, $x=F(x, y)$ and $y=F(y, x)$, that is, $F$ has a coupled fixed point. This completes the proof.

Corollary 3.2 Let $(X, \preceq)$ be a partially ordered set and $\mathcal{B}$ be a cone ball-metric induced by the cone metric $d$ on $X$ with a regular cone $P$ such that $(X, \mathcal{B})$ is a complete cone ball-metric space. Let $F: X \times X \rightarrow X$ be a continuous mapping having the mixed monotone property on $X$. Suppose that there exists $k \in[0,1)$ such that

$$
\begin{aligned}
& \mathcal{B}(F(x, y), F(u, v), F(w, z))+\mathcal{B}(F(y, x), F(v, u), F(z, w)) \\
& \quad \leq_{E} k(\mathcal{B}(x, u, w)+\mathcal{B}(y, v, z))
\end{aligned}
$$

for all $x, y, z, u, v, w \in X$ for which $x \succeq u \succeq w$ and $y \preceq v \preceq z$. If there exists $x_{0}, y_{0} \in X$ such that

$$
x_{0} \preceq F\left(x_{0}, y_{0}\right) \quad \text { and } \quad y_{0} \succeq F\left(y_{0}, x_{0}\right) \text {, }
$$

then $F$ has a coupled fixed point, that is, there exist $x, y \in X$ such that

$$
x=F(x, y) \text { and } y=F(y, x) .
$$

In the next theorem, we omit the continuity hypothesis of $F$.

Theorem 3.3 Let $(X, \preceq)$ be a partially ordered set and $\mathcal{B}$ be a cone ball-metric induced by the cone metric $d$ on $X$ with a regular cone $P$ such that $(X, \mathcal{B})$ is a complete cone ball-metric space. Let $F: X \times X \rightarrow X$ be a mapping having the mixed monotone property on $X$. Suppose that there exists $\delta \in \Delta$ such that

$$
\begin{gathered}
\mathcal{B}(F(x, y), F(u, v), F(w, z))+\mathcal{B}(F(y, x), F(v, u), F(z, w)) \\
\quad \leq_{E} \delta(\mathcal{B}(x, u, w), \mathcal{B}(y, v, z))(\mathcal{B}(x, u, w)+\mathcal{B}(y, v, z))
\end{gathered}
$$

for all $x, y, z, u, v, w \in X$ for which $x \succeq u \succeq w$ and $y \preceq v \preceq z$. If there exists $x_{0}, y_{0} \in X$ such that

$$
x_{0} \preceq F\left(x_{0}, y_{0}\right) \text { and } y_{0} \succeq F\left(y_{0}, x_{0}\right)
$$

and $X$ has the following property: 
(i) if a nondecreasing sequence $\left\{x_{n}\right\}$ converges to $x$, then $x_{n} \preceq x$ for all $n \in \mathbb{N}$,

(ii) if a nonincreasing sequence $\left\{y_{n}\right\}$ converges to $y$, then $y \geq y_{n}$ for all $n \in \mathbb{N}$,

then $F$ has a coupled fixed point, that is, there exist $x, y \in X$ such that

$$
x=F(x, y) \text { and } y=F(y, x) .
$$

Proof By the similar the proof as in Theorem 3.1, we have the nondecreasing sequence $\left\{x_{n}\right\}$ converges to $x$ and the nonincreasing sequence $\left\{y_{n}\right\}$ converges to $y$ for some $x, y \in X$. By (i), (ii), we get $x_{n} \preceq x$ and $y_{n} \succeq y$ for all $n \in \mathbb{N}$. Thus, by the rectangle inequality of $\mathcal{B}$, we get

$$
\begin{array}{rl}
\mathcal{B}(F(x, y), x, x)+\mathcal{B}(F(y, x), y, y) \\
\leq_{E} & \mathcal{B}\left(F(x, y), F\left(x_{n}, y_{n}\right), F\left(x_{n}, y_{n}\right)\right)+\mathcal{B}\left(F\left(x_{n}, y_{n}\right), x, x\right) \\
+ & \mathcal{B}\left(F(y, x), F\left(y_{n}, x_{n}\right), F\left(y_{n}, x_{n}\right)\right)+\mathcal{B}\left(F\left(y_{n}, x_{n}\right), y, y\right) \\
= & \mathcal{B}\left(F(x, y), F\left(x_{n}, y_{n}\right), F\left(x_{n}, y_{n}\right)\right)+\mathcal{B}\left(F(y, x), F\left(y_{n}, x_{n}\right), F\left(y_{n}, x_{n}\right)\right) \\
+ & \mathcal{B}\left(F\left(x_{n}, y_{n}\right), x, x\right)+\mathcal{B}\left(F\left(y_{n}, x_{n}\right), y, y\right) \\
\leq_{E} & \delta\left(\mathcal{B}\left(x, x_{n}, x_{n}\right), \mathcal{B}\left(y, y_{n}, y_{n}\right)\right)\left[\mathcal{B}\left(x, x_{n}, x_{n}\right)+\mathcal{B}\left(y, y_{n}, y_{n}\right)\right] \\
& +\mathcal{B}\left(x_{n+1}, x, x\right)+\mathcal{B}\left(y_{n+1}, y, y\right) \\
<_{E} & {\left[\mathcal{B}\left(x, x_{n}, x_{n}\right)+\mathcal{B}\left(y, y_{n}, y_{n}\right)\right]+\mathcal{B}\left(x_{n+1}, x, x\right)+\mathcal{B}\left(y_{n+1}, y, y\right) .}
\end{array}
$$

Taking the limit as $n \rightarrow \infty$, we have $\mathcal{B}(F(x, y), x, x)+\mathcal{B}(F(y, x), y, y)=0_{E}$, and thus $x=F(x, y)$ and $y=F(y, x)$. Therefore, $F$ has a coupled fixed point $(x, y)$ in $X \times X$. This completes the proof.

Corollary 3.4 Let $(X, \preceq)$ be a partially ordered set and $\mathcal{B}$ be a cone ball-metric induced by the cone metric $d$ on $X$ with a regular cone $P$ such that $(X, \mathcal{B})$ is a complete cone ball-metric space. Let $F: X \times X \rightarrow X$ be a mapping having the mixed monotone property on $X$. Suppose that there exists $k \in[0,1)$ such that

$$
\begin{aligned}
& \mathcal{B}(F(x, y), F(u, v), F(w, z))+\mathcal{B}(F(y, x), F(v, u), F(z, w)) \\
& \quad \leq_{E} k(\mathcal{B}(x, u, w)+\mathcal{B}(y, v, z))
\end{aligned}
$$

for all $x, y, z, u, v, w \in X$ for which $x \succeq u \succeq w$ and $y \preceq v \preceq z$. If there exists $x_{0}, y_{0} \in X$ such that

$$
x_{0} \preceq F\left(x_{0}, y_{0}\right) \text { and } y_{0} \succeq F\left(y_{0}, x_{0}\right)
$$

and $X$ has the following property:

(i) if a nondecreasing sequence $\left\{x_{n}\right\}$ converges to $x$, then $x_{n} \preceq x$ for all $n \in \mathbb{N}$,

(ii) if a nonincreasing sequence $\left\{y_{n}\right\}$ converges to $y$, then $y \geq y_{n}$ for all $n \in \mathbb{N}$, 
then $F$ has a coupled fixed point, that is, there exist $x, y \in X$ such that

$$
x=F(x, y) \text { and } y=F(y, x) .
$$

Theorem 3.5 In addition to the hypotheses in Theorem 3.1, suppose that $x_{0}, y_{0}$ are comparable then $x=y$, that is, $x=F(x, x)$.

Proof From Theorem 3.1, we have two sequences $\left\{x_{n}\right\},\left\{y_{n}\right\}$ in $X$ such that, for all $n \geq 0$,

$$
x_{n+1}=F\left(x_{n}, y_{n}\right) \quad \text { and } \quad y_{n+1}=F\left(y_{n}, x_{n}\right)
$$

and also $x_{n} \rightarrow x$ and $y_{n} \rightarrow y$ as $n \rightarrow \infty$. Now, we assume that $x_{0} \preceq y_{0}$. Since $F$ has the mixed monotone property, we have $x_{n} \preceq y_{n}$ for all $n \in \mathbb{N}$. From (3.1) and property of coneball metric $\mathcal{B}$, we have

$$
\begin{array}{rl}
2 & \mathcal{B}\left(y_{n+1}, x_{n+1}, x_{n+1}\right) \\
& =\mathcal{B}\left(y_{n+1}, x_{n+1}, x_{n+1}\right)+\mathcal{B}\left(x_{n+1}, y_{n+1}, y_{n+1}\right) \\
& =\mathcal{B}\left(F\left(y_{n}, x_{n}\right), F\left(x_{n}, y_{n}\right), F\left(x_{n}, y_{n}\right)\right)+\mathcal{B}\left(F\left(x_{n}, y_{n}\right), F\left(y_{n}, x_{n}\right), F\left(y_{n}, x_{n}\right)\right) \\
& \leq \\
& \delta\left(\mathcal{B}\left(y_{n}, x_{n}, x_{n}\right), \mathcal{B}\left(x_{n}, y_{n}, y_{n}\right)\right)\left[\mathcal{B}\left(y_{n}, x_{n}, x_{n}\right)+\mathcal{B}\left(x_{n}, y_{n}, y_{n}\right)\right] \\
& =\delta\left(\mathcal{B}\left(y_{n}, x_{n}, x_{n}\right), \mathcal{B}\left(y_{n}, x_{n}, x_{n}\right)\right)\left[2 \mathcal{B}\left(y_{n}, x_{n}, x_{n}\right)\right] .
\end{array}
$$

This implies

$$
\begin{aligned}
\mathcal{B}\left(y_{n+1}, x_{n+1}, x_{n+1}\right) & \leq_{E} \delta\left(\mathcal{B}\left(y_{n}, x_{n}, x_{n}\right), \mathcal{B}\left(y_{n}, x_{n}, x_{n}\right)\right)\left[\mathcal{B}\left(y_{n}, x_{n}, x_{n}\right)\right] \\
& <_{E} \mathcal{B}\left(y_{n}, x_{n}, x_{n}\right) .
\end{aligned}
$$

So we have $\left\{\mathcal{B}\left(y_{n}, x_{n}, x_{n}\right)\right\}$ is a decreasing sequence. Similar to the prove in Theorem 3.1, we get $\mathcal{B}\left(y_{n}, x_{n}, x_{n}\right) \rightarrow 0_{E}$ as $n \rightarrow \infty$.

By the rectangular inequality and (3.21), we have

$$
\begin{aligned}
\mathcal{B}(y, x, x) & \leq{ }_{E} \mathcal{B}\left(y, y_{n+1}, y_{n+1}\right)+\mathcal{B}\left(y_{n+1}, x_{n+1}, x_{n+1}\right)+\mathcal{B}\left(x_{n+1}, x, x\right) \\
& { }_{E} \mathcal{B}\left(y, y_{n+1}, y_{n+1}\right)+\mathcal{B}\left(y_{n}, x_{n}, x_{n}\right)+\mathcal{B}\left(x_{n+1}, x, x\right) .
\end{aligned}
$$

From above inequality, taking $n \rightarrow \infty$, we obtain that $\mathcal{B}(y, x, x)=0_{E}$ and then $x=y$. This completes the proof.

Theorem 3.6 In addition to the hypotheses in Theorem 3.3, suppose that $x_{0}, y_{0}$ are comparable then $x=y$, that is, $x=F(x, x)$.

Proof By the similar method as in the prove of Theorem 3.5 and by applying Theorem 3.3, we can get the conclusion.

Theorem 3.7 Let $(X, \preceq)$ be a partially ordered set and $\mathcal{B}$ be a cone ball-metric induced by the cone metric $d$ on $X$ with a regular cone $P$ such that $(X, \mathcal{B})$ is a complete cone ball-metric 
space. Let $F: X \times X \rightarrow X$ be a mapping having the mixed monotone property on $X$. Suppose that there exists $\vartheta \in \Delta$ such that

$$
\mathcal{B}(F(x, y), F(u, v), F(w, z)) \leq_{E} \frac{1}{2} \vartheta(\mathcal{B}(x, u, w), \mathcal{B}(y, v, z))(\mathcal{B}(x, u, w)+\mathcal{B}(y, v, z))
$$

for all $x, y, z, u, v, w \in X$ for which $x \succeq u \succeq w$ and $y \preceq v \preceq z$. If there exists $x_{0}, y_{0} \in X$ such that

$$
x_{0} \preceq F\left(x_{0}, y_{0}\right) \text { and } y_{0} \succeq F\left(y_{0}, x_{0}\right)
$$

and either

(a) $F$ is continuous or

(b) $X$ has the following property:

(i) if a nondecreasing sequence $\left\{x_{n}\right\}$ converges to $x$, then $x_{n} \preceq x$ for all $n \in \mathbb{N}$,

(ii) if a nonincreasing sequence $\left\{y_{n}\right\}$ converges to $y$, then $y \succeq y_{n}$ for all $n \in \mathbb{N}$, then $F$ has a coupled fixed point.

Proof For any $x, y, z, u, v, w \in X$ with $x \succeq u \succeq w$ and $y \preceq v \preceq z$, it follows from (3.22) that

$$
\mathcal{B}(F(x, y), F(u, v), F(w, z)) \leq_{E} \frac{1}{2} \vartheta(\mathcal{B}(x, u, w), \mathcal{B}(y, v, z))(\mathcal{B}(x, u, w)+\mathcal{B}(y, v, z))
$$

and

$$
\begin{aligned}
\mathcal{B}(F(y, x), F(v, u), F(z, w)) & =\mathcal{B}(F(z, w), F(v, u), F(y, x)) \\
& \leq E \frac{1}{2} \vartheta(\mathcal{B}(z, v, y), \mathcal{B}(w, u, x))(\mathcal{B}(z, v, y)+\mathcal{B}(w, u, x)) \\
& =\frac{1}{2} \vartheta(\mathcal{B}(z, v, y), \mathcal{B}(w, u, x))(\mathcal{B}(x, u, w), \mathcal{B}(y, v, z)) .
\end{aligned}
$$

From (3.23) and (3.24), we have

$$
\begin{aligned}
\mathcal{B}( & F(x, y), F(u, v), F(w, z))+\mathcal{B}(F(y, x), F(v, u), F(z, w)) \\
\quad & \leq_{E} \frac{1}{2}[\vartheta(\mathcal{B}(x, u, w), \mathcal{B}(y, v, z))+\vartheta(\mathcal{B}(z, v, y), \mathcal{B}(w, u, x))](\mathcal{B}(x, u, w)+\mathcal{B}(y, v, z)) \\
& =\delta(\mathcal{B}(x, u, w), \mathcal{B}(y, v, z)))(\mathcal{B}(x, u, w)+\mathcal{B}(y, v, z))
\end{aligned}
$$

for all $x, y, z, u, v, w \in X$ with $x \succeq u \succeq w$ and $y \preceq v \preceq z$, where

$$
\delta(a, b)=\frac{1}{2}[\vartheta(a, b)+\vartheta(b, a)]
$$

for all $a, b \in \operatorname{int}(P) \cup\left\{0_{E}\right\}$. It is easy to verify that $\delta \in \Delta$. If we apply Theorems 3.1 and 3.3, we know that $F$ has a coupled fixed point.

Corollary 3.8 Let $(X, \preceq)$ be a partially ordered set and $\mathcal{B}$ be a cone ball-metric induced by the cone metric $d$ on $X$ with a regular cone $P$ such that $(X, \mathcal{B})$ is a complete cone ball-metric 
space. Let $F: X \times X \rightarrow X$ be a mapping having the mixed monotone property on $X$. Suppose that there exists $k \in[0,1)$ such that

$$
\mathcal{B}(F(x, y), F(u, v), F(w, z)) \leq_{E} \frac{k}{2}(\mathcal{B}(x, u, w)+\mathcal{B}(y, v, z))
$$

for all $x, y, z, u, v, w \in X$ for which $x \succeq u \succeq w$ and $y \preceq v \preceq z$. If there exists $x_{0}, y_{0} \in X$ such that

$$
x_{0} \preceq F\left(x_{0}, y_{0}\right) \quad \text { and } \quad y_{0} \succeq F\left(y_{0}, x_{0}\right)
$$

and either

(a) $F$ is continuous or

(b) $X$ has the following property:

(i) if a nondecreasing sequence $\left\{x_{n}\right\}$ converges to $x$, then $x_{n} \preceq x$ for all $n \in \mathbb{N}$,

(ii) if a nonincreasing sequence $\left\{y_{n}\right\}$ converges to $y$, then $y \succeq y_{n}$ for all $n \in \mathbb{N}$,

then $F$ has a coupled fixed point.

Let $\Xi$ denote the class of functions $\xi: \operatorname{int}(P) \cup\left\{0_{E}\right\} \rightarrow[0,1)$ which satisfies the following condition:

For any sequence $\left\{a_{n}\right\} \operatorname{in} \operatorname{int}(P) \cup\left\{0_{E}\right\}$,

$$
\xi\left(a_{n}\right) \rightarrow 1 \quad \Longrightarrow \quad a_{n} \rightarrow 0_{E}
$$

Theorem 3.9 Let $(X, \preceq)$ be a partially ordered set and $\mathcal{B}$ be a cone ball-metric induced by the cone metric $d$ on $X$ with a regular cone $P$ such that $(X, \mathcal{B})$ is a complete cone ball-metric space. Let $F: X \times X \rightarrow X$ be a mapping having the mixed monotone property on $X$. Suppose that there exists $\xi \in \Xi$ such that

$$
\begin{gathered}
\mathcal{B}(F(x, y), F(u, v), F(w, z))+\mathcal{B}(F(y, x), F(v, u), F(z, w)) \\
\quad \leq_{E} \xi(\mathcal{B}(x, u, w)+\mathcal{B}(y, v, z))(\mathcal{B}(x, u, w)+\mathcal{B}(y, v, z))
\end{gathered}
$$

for all $x, y, z, u, v, w \in X$ for which $x \succeq u \succeq w$ and $y \preceq v \preceq z$ If there exists $x_{0}, y_{0} \in X$ such that

$$
x_{0} \preceq F\left(x_{0}, y_{0}\right) \text { and } y_{0} \succeq F\left(y_{0}, x_{0}\right)
$$

and either

(a) $F$ is continuous or

(b) $X$ has the following property:

(i) if a nondecreasing sequence $\left\{x_{n}\right\}$ converges to $x$, then $x_{n} \preceq x$ for all $n \in \mathbb{N}$,

(ii) if a nonincreasing sequence $\left\{y_{n}\right\}$ converges to $y$, then $y \succeq y_{n}$ for all $n \in \mathbb{N}$,

then $F$ has a coupled fixed point.

Proof If we taking $\delta(a, b)=\xi(a+b)$ for all $a, b \in \operatorname{int}(P) \cup\left\{0_{E}\right\}$ in Theorem 3.1, then, from (a), we get the conclusion. Also, if we take $\delta(a, b)=\xi(a+b)$ for all $a, b \in \operatorname{int}(P) \cup\left\{0_{E}\right\}$ in Theorem 3.3, then, from (b), we obtain the conclusion. 
Theorem 3.10 Let $(X, \preceq)$ be a partially ordered set and $\mathcal{B}$ be a cone ball-metric induced by the cone metric $d$ on $X$ with a regular cone $P$ such that $(X, \mathcal{B})$ is a complete cone ballmetric space. Let $F: X \times X \rightarrow X$ be a mapping having the mixed monotone property on $X$ and such that $F(x, y) \preceq F(y, x)$, whenever $x \preceq y$. Suppose that there exists $\delta \in \Delta$ such that

$$
\begin{gathered}
\mathcal{B}(F(x, y), F(u, v), F(w, z))+\mathcal{B}(F(y, x), F(v, u), F(z, w)) \\
\quad \leq_{E} \delta(\mathcal{B}(x, u, w), \mathcal{B}(y, v, z))(\mathcal{B}(x, u, w)+\mathcal{B}(y, v, z))
\end{gathered}
$$

for all $x, y, z, u, v, w \in X$ for which $w \preceq u \preceq x \prec y \preceq v \preceq z$. If there exists $x_{0}, y_{0} \in X$ such that

$$
x_{0} \preceq y_{0}, \quad x_{0} \preceq F\left(x_{0}, y_{0}\right) \quad \text { and } \quad y_{0} \succeq F\left(y_{0}, x_{0}\right)
$$

and either

(a) $F$ is continuous or

(b) $X$ has the following property:

(i) if a nondecreasing sequence $\left\{x_{n}\right\}$ converges to $x$, then $x_{n} \preceq x$ for all $n \in \mathbb{N}$,

(ii) if a nonincreasing sequence $\left\{y_{n}\right\}$ converges to $y$, then $y \geq y_{n}$ for all $n \in \mathbb{N}$,

then $F$ has a coupled fixed point.

Proof From the assumption, there exist $x_{0}, y_{0} \in X$ such that $x_{0} \preceq F\left(x_{0}, y_{0}\right)$ and $y_{0} \succeq$ $F\left(y_{0}, x_{0}\right)$. Now, we define $x_{1}, y_{1} \in X$ such that $x_{1}=F\left(x_{0}, y_{0}\right) \succeq x_{0}$ and $y_{1}=F\left(y_{0}, x_{0}\right) \preceq y_{0}$. Further, the fact $x_{0} \preceq y_{0}$, we get $F\left(x_{0}, y_{0}\right) \preceq F\left(y_{0}, x_{0}\right)$. Thus, we now have

$$
x_{0} \preceq x_{1}=F\left(x_{0}, y_{0}\right) \preceq F\left(y_{0}, x_{0}\right)=y_{1} \preceq y_{0} .
$$

Let $x_{2}=F\left(x_{1}, y_{1}\right)$ and $y_{2}=F\left(y_{1}, x_{1}\right)$. From the fact that $x_{1} \preceq y_{1}$ and the mixed monotone property, we have

$$
x_{1} \preceq x_{2} \preceq y_{2} \preceq y_{1} .
$$

Continuing this procedure, we have two sequences $\left\{x_{n}\right\}$ and $\left\{y_{n}\right\}$ such that

$$
x_{n+1}=F\left(x_{n}, y_{n}\right), \quad y_{n+1}=F\left(y_{n}, x_{n}\right)
$$

and

$$
x_{n} \preceq x_{n+1}=F\left(x_{n}, y_{n}\right) \preceq F\left(y_{n}, x_{n}\right)=y_{n+1} \preceq y_{n}
$$

for all $n \geq 0$. If there exists a nonnegative integer $k$ such that $x_{k}=y_{k}=c$ (say), then we have

$$
c \preceq F(c, c) \preceq F(c, c) \preceq c,
$$

that is, $c=F(c, c)$. Therefore, $(c, c)$ is a coupled fixed point of $F$.

Therefore, we assume that

$$
x_{n} \prec y_{n}
$$


for all $n \geq 0$. In view of (3.29), for all $n \geq 0$, the inequality (3.28) holds with

$$
\begin{aligned}
& w=x_{n}, \quad u=x_{n+1}, \quad x=x_{n+1}, \\
& y=y_{n+1}, \quad v=y_{n+1} \quad \text { and } \quad z=y_{n} .
\end{aligned}
$$

The rest of the proof can be completed by repeating the same steps given in Theorem 3.1 and Theorem 3.3. This completes the proof.

Example 3.11 Let $E=\mathbb{R}$ be a usual norm space with a regular cone $P=\{z \in \mathbb{R} \mid z \geq 0\}$ and $X=\mathbb{N} \cup\{0\}$ be a cone metric space with a metric $d(x, y)=|x-y|$ for all $x, y \in X$. Then $(X, d)$ is a complete cone metric space. Therefore, we get a cone ball metric $\mathcal{B}: X \times X \times X \rightarrow E$ such that

$$
\mathcal{B}(x, y, z)=\max \{x, y, z\}-\min \{x, y, z\}
$$

for all $x, y, z \in X$ and so $(X, \mathcal{B})$ is a complete cone ball-metric space.

Let a partial order $\preceq$ on $X$ be defined as follows: For $x, y \in X$,

$$
x \preceq y \text { holds if } x \geq_{E} y .
$$

Let $F: X \times X \rightarrow X$ be defined by

$$
F(x, y)= \begin{cases}1 ; & \text { if } x \prec y, \\ 0 ; & \text { if otherwise }\end{cases}
$$

Let $w \preceq u \preceq x \prec y \preceq v \preceq z$ hold, then we have $w \geq_{E} u \geq_{E} x>_{E} y \geq_{E} v \geq_{E} z$. Therefore, we have

$$
F(x, y)=F(u, v)=F(w, z)=1
$$

and

$$
F(y, x)=F(v, u)=F(z, w)=0 .
$$

So the left side of (3.28) is

$$
\mathcal{B}(1,1,1)+\mathcal{B}(0,0,0)=0
$$

and then (3.28) is satisfied for all $\delta \in \Delta$. Thus, Theorem 3.10 is applicable to this example with $x_{0}=81$ and $y_{0}=0$. Therefore, $F$ has a coupled fixed points that is a point $(0,0)$ and $(1,0)$.

Remark 3.12 Example 3.11 is not applied by Theorems 3.1,3.3, and 3.9. This is evident by the fact that the inequality (3.1), (3.19), and (3.27) are not satisfied when $w=u=x=y=3$, $v=0$ and $z=1$. Moreover, the coupled fixed point is not unique. 


\section{Uniqueness of coupled fixed point in cone ball-metric spaces}

In this section, we study the necessary condition for the uniqueness of a coupled fixed point. If $(X, \preceq)$ is a partially ordered set, then we endow the product of $X \times X$ with the following partial order relation: for any $(x, y),(u, v) \in X \times X$,

$$
(x, y) \lessdot(u, v) \Longleftrightarrow x \preceq u \text { and } y \succeq v \text {. }
$$

Theorem 4.1 In addition to the hypotheses in Theorem 3.1, suppose that, for any $(x, y)$, $(z, t) \in X \times X$, there exists a point $(u, v) \in X \times X$ which is comparable to $(x, y)$ and $(z, t)$. Then $F$ has a unique coupled fixed point.

Proof By Theorem 3.1, we get $F$ has a coupled fixed point $(x, y)$, that is,

$$
x=F(x, y) \text { and } y=F(y, x) .
$$

We may assume that $(z, t)$ are another coupled fixed points of $F$ and so

$$
z=F(z, t) \text { and } \quad t=F(t, z) \text {. }
$$

Next, we prove that $x=z$ and $y=t$. By assumption, there exists $(u, v) \in X \times X$ which is comparable to $(x, y)$ and $(z, t)$. We put $u_{0}=u$ and $v_{0}=v$ and construct two sequences $\left\{u_{n}\right\}$ and $\left\{v_{n}\right\}$ by

$$
u_{n}=F\left(u_{n-1}, v_{n-1}\right) \quad \text { and } \quad v_{n}=F\left(v_{n-1}, u_{n-1}\right)
$$

for all $n \in \mathbb{N}$. Since $(u, v)$ is comparable with $(x, y)$, we may assume that $\left(u_{0}, v_{0}\right)=(u, v) \lessdot$ $(x, y)$. It easy to see that $\left(u_{n}, v_{n}\right) \lessdot(x, y)$ for all $n \in \mathbb{N}$. From (3.1), we have

$$
\begin{aligned}
\mathcal{B}\left(x, x, u_{n}\right)+\mathcal{B}\left(y, y, v_{n}\right) \\
\quad=\mathcal{B}\left(F(x, y), F(x, y), F\left(u_{n-1}, v_{n-1}\right)\right)+\mathcal{B}\left(F(y, x), F(y, x), F\left(v_{n-1}, u_{n-1}\right)\right) \\
\quad \leq_{E} \delta\left(\mathcal{B}\left(x, x, u_{n-1}\right), \mathcal{B}\left(v_{n-1}, y, y\right)\right)\left[\mathcal{B}\left(x, x, u_{n-1},\right)+\mathcal{B}\left(v_{n-1}, y, y\right)\right] \\
\quad{ }_{E} \mathcal{B}\left(x, x, u_{n-1}\right)+\mathcal{B}\left(v_{n-1}, y, y\right) .
\end{aligned}
$$

This implies that $\left\{\mathcal{B}\left(x, x, u_{n}\right)+\mathcal{B}\left(y, y, v_{n}\right)\right\}$ is a decreasing sequence and so

$$
\mathcal{B}\left(x, x, u_{n}\right)+\mathcal{B}\left(y, y, v_{n}\right) \rightarrow \mathcal{B}
$$

for some $\mathcal{B} \geq_{E} 0_{E}$.

Now, we show that $\mathcal{B}=0_{E}$. We may assume that $\mathcal{B}>_{E} 0_{E}$. By the similar method as in the proof of Theorem 3.1, we can conclude that

$$
\delta\left(\mathcal{B}\left(x, x, u_{n-1}\right), \mathcal{B}\left(v_{n-1}, y, y\right)\right) \rightarrow 1 .
$$

Since $\delta \in \Delta$, we get $\mathcal{B}\left(x, x, u_{n-1}\right) \rightarrow 0_{E}$ and $\mathcal{B}\left(v_{n-1}, y, y\right) \rightarrow 0_{E}$. Therefore, we have

$$
\mathcal{B}\left(x, x, u_{n-1}\right)+\mathcal{B}\left(v_{n-1}, y, y\right) \rightarrow 0_{E},
$$


which is a contradiction. Thus, we have $\mathcal{B}\left(x, x, u_{n}\right)+\mathcal{B}\left(v_{n}, y, y\right) \rightarrow 0_{E}$ as $n \rightarrow \infty$. Similarly, one can prove $\mathcal{B}\left(z, z, u_{n}\right)+\mathcal{B}\left(v_{n}, t, t\right) \rightarrow 0_{E}$ as $n \rightarrow \infty$.

Finally, we have

$$
\begin{aligned}
\mathcal{B}(z, x, x)+\mathcal{B}(y, t, t) & \leq_{E} \mathcal{B}\left(z, u_{n}, u_{n}\right)+\mathcal{B}\left(u_{n}, x, x\right)+\mathcal{B}\left(y, v_{n}, v_{n}\right)+\mathcal{B}\left(v_{n}, t, t\right) \\
& =\mathcal{B}\left(z, z, u_{n}\right)+\mathcal{B}\left(x, x, u_{n}\right)+\mathcal{B}\left(y, y, v_{n}\right)+\mathcal{B}\left(v_{n}, t, t\right) .
\end{aligned}
$$

Taking $n \rightarrow \infty$ in above inequalities, we have $\mathcal{B}(z, x, x)+\mathcal{B}(y, t, t)=0_{E}$, that is, $z=x$ and $y=t$.

For the case when $\left(u_{0}, v_{0}\right)=(u, v)>(x, y)$ is similar. This completes the proof.

Theorem 4.2 In addition to the hypotheses in Theorem 3.3, suppose that, for any $(x, y),(z, t) \in X \times X$, there exists a point $(u, v) \in X \times X$ which is comparable to $(x, y)$ and $(z, t)$. Then $F$ has a unique coupled fixed point.

Proof By the similar method given in the prove of Theorem 4.1 and by applying Theorem 3.3, we can get the conclusion.

\section{Open problems:}

- In our theorems, can the mixed monotone property be replaced by a more general property (see the work of Sintunavarat et al. [36])?

- In our theorems, can the mixed monotone property be replaced by another property (see the work of Đorić et al. [16])?

- Can the coupled fixed-point theorems in this paper be extended to coupled best proximity point theorems (see the work of Sintunavarat et al. [37])?

- Can the main results in this paper be extended to multivalued case of coupled fixed point?

- Can the concept of cone ball-metric be extended to another distance?

\section{Competing interests}

The authors declare that they have no competing interests.

\section{Authors' contributions}

All authors read and approved the final manuscript.

\section{Acknowledgements \\ The first author would like to thank the Research Professional Development Project under the Science Achievement Scholarship of Thailand (SAST), the second author was supported by the Basic Science Research Program through the National Research Foundation of Korea (NRF) funded by the Ministry of Education, Science, and Technology (Grant No. 2011-0021821), and the third author was supported by the Commission on Higher Education, the Thailand Research Fund and the King Mongkut's University of Technology Thonburi (Grant No. MRG5580213) for financial support during the preparation of this manuscript.}

Received: 11 April 2012 Accepted: 19 July 2012 Published: 3 August 2012

\section{References}

1. Abbas, M, Jungck, G: Common fixed point results for noncommuting mappings without continuity in cone metric space. J. Math. Anal. Appl. 341, 416-420 (2008)

2. Abbas, M, Rhoades, BE: Fixed and periodic point results in cone metric spaces. Appl. Math. Lett. 22, $511-515$ (2009)

3. Abbas, M, Ali Khan, M, Radenovic, S: Common coupled fixed point theorems in cone metric spaces for $w$-compatible mappings. Appl. Math. Comput. 217(1), 195-202 (2010)

4. Abbas, M, Khan, AR, Nazir, T: Coupled common fixed point results in two generalized metric spaces. Appl. Math Comput. 17(13), 6328-6336 (2011)

5. Abbas, M, Sintunavarat, W, Kumam, P: Coupled fixed point of generalized contractive mappings on partially ordered G-metric spaces. Fixed Point Theory Appl. 2012, 31 (2012) 
6. Altun, I, Simsek, H: Some fixed point theorems on ordered metric spaces and application. Fixed Point Theory Appl. 2010, Article ID 621469 (2010)

7. Arvanitakis, AD: A proof of the generalized Banach contraction conjecture. Proc. Am. Math. Soc. 131(12), $3647-3656$ (2003)

8. Aydi, H, Damjanovic, B, Samet, B, Shatanawi, W: Coupled fixed point theorems for nonlinear contractions in partially ordered G-metric spaces. Math. Comput. Model. 54, 2443-2450 (2011)

9. Aydi, H, Vetro, C, Sintunavarat, W, Kumam, P: Coincidence and fixed points for contractions and cyclical contractions in partial metric spaces. Fixed Point Theory Appl. 2012, 124 (2012)

10. Azam, A, Arshad, M: Common fixed points of generalized contractive maps in cone metric space. Bull. Iran. Math. Soc. 35(2), 225-264 (2009)

11. Banach, S: Sur les opérations dans les ensembles abstraits et leurs applications aux équations intégrales. Fundam. Math. 3, 133-181 (1922)

12. Bhaskar, TG, Lakshmikantham, V: Fixed point theorems in partially ordered metric spaces and applications. Nonlinear Anal. 65, 1379-1393 (2006)

13. Boyd, DW, Wong, JSW: On nonlinear contractions. Proc. Am. Math. Soc. 20, 458-464 (1969)

14. Chen, CM, Chang, TH, Juang, KS: Common fixed point theorems for the stronger Meir-Keeler cone-type function in cone ball-metric spaces. Appl. Math. Lett. 25, 692-697 (2012)

15. Choudhury, BS, Das, KP: A new contraction principle in Menger spaces. Acta Math. Sin. 24(8), 1379-1386 (2008)

16. Đorić, D, Kadelburg, Z, Radenović, S: Coupled fixed point results for mappings without mixed monotone property. Appl. Math. Lett. (2012). doi:10.1016/j.aml.2012.02.022

17. Harjani, J, Sadarangani, K: Generalized contractions in partially ordered metric spaces and applications to ordinary differential equations. Nonlinear Anal. 72, 1188-1197 (2010)

18. Huang, L-G, Zhang, X: Cone metric spaces and fixed point theorems of contractive mappings. J. Math. Anal. Appl. $332,1468-1476(2007)$

19. Jungck, G, Radenović, S, Radojević, S, Rakočević, V: Common fixed point theorems for weakly compatible pairs on cone metric spaces. Fixed Point Theory Appl. 2009, Article ID 643840 (2009)

20. Ilić, D, Rakočević, V: Common fixed point for maps on cone metric space. J. Math. Anal. Appl. 341, 876-882 (2008)

21. Ilić, D, Rakočević, V: Quasi-contraction on a cone metric space. Appl. Math. Lett. 22, 728-731 (2009)

22. Lakshmikantham, V, Ciric, L: Coupled fixed point theorems for nonlinear contractions in partially ordered metric spaces. Nonlinear Anal., Theory Methods Appl. 70(12), 4341-4349 (2009)

23. Luong, NV, Thuan, NX: Coupled fixed point theorems in partially ordered G-metric spaces. Math. Comput. Model. 55(3-4), 1601-1609 (2012)

24. Mongkolkeha, C, Kumam, P: Fixed point and common fixed point theorems for generalized weak contraction mappings of integral type in modular spaces. Int. J. Math. Math. Sci. 2011, Article ID 705943 (2011)

25. Nieto, JJ, Lopez, RR: Existence and uniqueness of fixed point in partially ordered sets and applications to ordinary differential equations. Acta Math. Sin. Engl. Ser. 23(12), 2205-2212 (2007)

26. Ran, ACM, Reurings, MCB: A fixed point theorem in partially ordered sets and some applications to matrix equations. Proc. Am. Math. Soc. 132, 1435-1443 (2004)

27. Rezapour, S, Hamlbarani, R: Some notes on paper cone metric spaces and fixed point theorems of contractive mappings. J. Math. Anal. Appl. 345, 719-724 (2008)

28. Samet, B, Vetro, C: Coupled fixed point theorems for multi-valued nonlinear contraction mappings in partially ordered metric spaces. Nonlinear Anal., Theory Methods Appl. 74(12), 4260-4268 (2011)

29. Shatanawi, W: Partially ordered cone metric spaces and coupled fixed point results. Comput. Math. Appl. 60(8), 2508-2515 (2010)

30. Shatanawi, W, Samet, B, Abbas, M: Coupled fixed point theorems for mixed monotone mappings in ordered partial metric spaces. Math. Comput. Model. 55(3-4), 680-687 (2012)

31. Sintunavarat, W, Kumam, P: Weak condition for generalized multi-valued $(f, \alpha, \beta)$-weak contraction mappings. Appl. Math. Lett. 24, 460-465 (2011)

32. Sintunavarat, W, Kumam, P: Gregus type fixed points for a tangential multi-valued mappings satisfying contractive conditions of integral type. J. Inequal. Appl. 2011, 3 (2011)

33. Sintunavarat, W, Cho, YJ, Kumam, P: Common fixed point theorems for c-distance in ordered cone metric spaces. Comput. Math. Appl. 62, 1969-1978 (2011)

34. Sintunavarat, W, Kumam, P: Common fixed point theorems for generalized $\mathcal{J} \mathcal{H}$-operator classes and invariant approximations. J. Inequal. Appl. 2011, 67 (2011)

35. Sintunavarat, W, Cho, YJ, Kumam, P: Coupled coincidence point theorems for contractions without commutative condition in intuitionistic fuzzy normed spaces. Fixed Point Theory Appl. 2011, 81 (2011)

36. Sintunavarat, W, Cho, YJ, Kumam, P: Coupled fixed point theorems for weak contraction mapping under F-invariant set. Abstr. Appl. Anal. 2012, Article ID 324874 (2012)

37. Sintunavarat, W, Kumam, P: Coupled best proximity point theorem in metric spaces. Fixed Point Theory Appl. 2012, $93(2012)$

38. Wardowski, D: Endpoint and fixed points of set-valued contractions in cone metric spaces. Nonlinear Anal. 71, 512-516 (2009)

doi:10.1186/1687-1812-2012-128

Cite this article as: Sintunavarat et al.: Coupled fixed-point theorems for contraction mapping induced by cone

ball-metric in partially ordered spaces. Fixed Point Theory and Applications 2012 2012:128. 